\title{
Agroindustria Rural y Mercados de Trabajo. ¿Alternativa a la Pobreza Rural?
}

\author{
Gerardo Torres Salcido*
}

Resumen: El debate sobre los mercados de trabajo rural ha abordado con amplitud las condiciones de la oferta y la demanda de fuerza de trabajo, de la segmentación y la exclusión de los grupos étnicos y de las mujeres en el campo. No obstante, poco se ha estudiado la relación entre la pobreza, las capacidades y la construcción de alternativas. En este artículo se explora esa relación por medio del Desarrollo Rural Territorial, de la apropiación de valor que se lleva a cabo a partir de las actividades agroindustriales rurales y de la construcción de Sistemas Agroalimentarios Localizados. A partir de la observación de algunos casos, se concluye que en las capacidades juegan un papel fundamental en la apropiación de valor, pero a condición de estar unidas al capital social, la gobernanza y la construcción de instituciones.

Palabras-clave: Pobreza Rural, Agroindustria Rural, Mercados de Trabajo, Sistemas Productivos Locales, Desarrollo Rural.

Abstract: The debate on the rural labour market has boarded with amplitude the conditions of the supply and demand of work force, of the segmentation and the exclusion of the ethnic groups and the women in the field. However, few are the studies on the relationship among poverty, capabilities and the construction of alternatives to rural poverty. In this article we explore that relation through the Local and Territorial Rural Development theory. We focus on the Rural Agro-Industry and the Local Agro Food Systems as instruments to valorise local products. From the observation of study cases we conclude that the capabilities play an important role in the appropriation of value and in the increase of income growth and well-being, but only on the condition that capabilities could be united to social capital, governance and institutional development.

Keywords: Rural Poverty, Rural Agro-Industry, Labor Markets, Productive Local Systems, Rural Development.

\footnotetext{
${ }^{*}$ Centro de Investigaciones Interdisciplinarias en Ciencias y Humanidades - Universidad Nacional Autónoma de México/ UNAM. E-mail: tsalcido@servidor.unam.mx. Recebido em 03/02/07 e aceito em 31/10/07.
} 


\section{Introducción}

La caracterización de los mercados de trabajo rurales ha tenido avances teóricos que se han realizado en un tiempo relativamente corto. En las últimas dos décadas se ha transitado de los estudios centrados en la oferta y la demanda de mano de obra, a los enfoques institucionales de los efectos de la reestructuración productiva, pasando por las investigaciones que han enfatizado el surgimiento de nuevos actores y nuevos intermediarios en el mercado de trabajo (APARICIO, BERENGUER, RAU, 2004; ASTORGA LIRA, 1985; BARRÓN PÉREZ, SIFUENTES, PEDRERO, 1997; GARZA TOLEDO, SALAS, 2000; LARA FLORES, 2001, p. 363-365; TORRES SALCIDO, 2005).

Aunque muchos de esos estudios reconocen que la precariedad, la reestructuración productiva en el campo y la marginación de los trabajadores tienen relación con la pobreza rural, existen pocos trabajos que abordan la pobreza desde el punto de vista de la carencia de capacidades. Asimismo, poco se ha reflexionado sobre la función de las capacidades para la construcción de sistemas de mercado alternativos, la diversificación de las fuentes de empleo y el uso de los recursos locales para a elevación del ingreso y el bienestar de los hogares rurales. Para emprender el conocimiento de esas relaciones, creemos imprescindible poner de relieve dos estrategias relativamente recientes de los hogares campesinos en el contexto Latinoamericano: la primera es el desarrollo territorial ${ }^{1}$ y la segunda, es la diversificación del empleo rural no agrícola.

El interés de este trabajo reside en contribuir al desarrollo del debate de los mercados de trabajo tomando en cuenta ambas perspectivas. El método que seguiremos en este artículo es distinguir conceptualmente el entramado institucional de los mercados de trabajo rurales, los que entendemos no sólo como la oferta y la demanda de mano de obra en condiciones segmentadas o excluyentes, sino como los lugares en los que se confeccionan las alternativas. En este artículo entenderemos por alternativas, la elaboración y consolidación

\footnotetext{
${ }^{1}$ Es necesario señalar que como todos los conceptos y categorías que se utilizan cotidianamente y en la medida que son incorporados en la definición de instrumentos de política, puede existir una tendencia a banalizar el territorio. De hecho, en las políticas a menudo se le menciona como un espacio delimitado geográficamente, pero se olvida el aspecto sociológico del mismo como un espacio socialmente construido, significado y resignificado incesantemente por las referencias de sus habitantes, con su cultura y sus instituciones específicas.
} 
de proyectos multifuncionales que son puestos en valor por los habitantes del territorio con base en sus capacidades ${ }^{2}$.

Las hipótesis que guiarán a este artículo son las siguientes:

- El grado de ventaja o desventaja de incorporarse a los mercados de trabajo dependerá de las capacidades. La escolaridad, la salud y la alimentación son la base de una serie de capacidades ampliadas, tales como el conocimiento de los derechos y la habilidad para desenvolverse en una comunidad. En este sentido, la existencia, creación, reconocimiento y consolidación de esas habilidades es la base para la generación y retención de valor en las comunidades rurales.

- La Agroindustria Rural (AIR) es la organización orientada a los procesos de postcosecha. Pero más allá de ello, representan el desarrollo de capacidades con una base territorial. La AIR ha sido fundamental para el funcionamiento de los actores campesinos en la reestructuración global de los sistemas agroalimentarios y en la defensa y valorización de los productos locales.

- Con base en experiencias de otras partes del mundo, pero sobre todo de Europa, es factible sostener que el desarrollo sustentable de la AIR y de los Sistemas Agroalimentarios Locales (SIAL) es posible a condición de que se transforme el consumo urbano, pero también en la medida que se consoliden los procesos de gobernanza territorial, que se traducen en el desarrollo de organizaciones locales, regionales y nacionales; en el diálogo, el consenso y en la sinergia institucional entre políticas, programas y organizaciones.

De acuerdo a lo anterior, en este trabajo plantearemos los siguientes objetivos:

- Reflexionar sobre el concepto de desarrollo rural con base al interés creciente en los territorios y en la multifuncionalidad de las unidades productivas.

- Definir la AIR y mostrar algunos ejemplos significativos.

- Establecer algunas bases para el desarrollo de programas de valorización de productos y de fuentes de empleo con base en el desarrollo de SIAL.

El método que seguiremos será el de un estudio exploratorio de la bibliografía y referencias específicas a estudios de caso. Para debatir estas

\footnotetext{
${ }^{2}$ Sen deja como presupuestas para el lector, en muchas ocasiones, la definición de las capacidades. Pero en Nuevo Examen de la Desigualdad, las aborda como expresión de la libertad de realización y no sólo como un acceso a bienes primarios. Esta definición sintetiza la idea de que la pobreza es ante todo falta de capacidad. Es decir, carencia de realizaciones por la imposibilidad de llevar a cabo elecciones. En sentido estricto, carencia de libertad.
} 
hipótesis y su viabilidad, dividiremos esta contribución en los siguientes apartados:

- La pobreza rural en México

- Las capacidades en el debate sobre la pobreza rural

- La definición de la AIR, su distinción respecto a otras formas de agroindustria y su importancia en la constitución de alternativas; y finalmente,

- La importancia de los SIAL y sus implicaciones para la propuesta de políticas.

\section{La pobreza rural en México. ¿Pobreza de qué?}

Los análisis tradicionales de la pobreza rural han concentrado su atención en la falta de ingreso, o bien en una carencia de capital humano (salud, educación, etcétera). Sin embargo, a partir de hace unos años, se ha establecido un vínculo entre pobreza y capacidades. El Comité Técnico de Medición de la Pobreza en México fija tres tipos de carencias. La primera de ellas es la alimentaria definida como el límite en el cual el consumo alimenticio de los hogares y las personas pone en riesgo su seguridad. El segundo nivel es el de la carencia de capacidades determinado como un insuficiente acceso a la educación y la salud. Finalmente, se encuentra, en el tercer nivel, la carencia de activos (propiedad, crédito, etcétera). Con base en las Encuestas Nacionales de Ingreso y Gasto de los Hogares (ENIGH) se caracterizan esos tipos de pobreza vinculadas al ingreso (COMITÉ TÉCNICO DE MEDICIÓN DE LA POBREZA, 2002).

Esta clasificación ha demostrado su utilidad para tipificar la pobreza en general, y la pobreza rural en particular. Asimismo, ha sido particularmente valiosa para el diseño e implementación de políticas públicas de combate a la pobreza. No obstante, desde el punto de vista del mercado y de la producción, uno de los problemas que presenta esta concepción es que las capacidades se muestran sólo como producto del ingreso debido a las restricciones de las fuentes oficiales de información. Ello introduce algunos problemas para definir las condicionantes de la pobreza. La primera forma de la pobreza, la carencia de ingreso suficiente para cubrir las necesidades alimenticias más urgentes, ha sido, por su propia naturaleza, la que más atención ha captado. Una de las críticas más amplias a la forma de medición de la pobreza con 
base en la deficiencia del consumo alimenticio de los pobres extremos es la visión asistencial que predomina en los programas. Sin embargo, aún al interior de los hogares con carencias alimenticias pueden existir decisiones que incrementan las capacidades. Una de ellas puede ser inscribirse en un programa social de combate a la pobreza como Oportunidades, en México que exige la permanencia en la escuela de los niños, mientras que otros miembros del hogar ven la migración como un recurso válido.

La segunda y tercera forma de la pobreza se desarrolla en términos de lo que comúnmente se conoce como una medida descomponible de la pobreza que permite observar la profundización de los niveles de la desigualdad entre los hogares pobres y los extremadamente pobres (FOSTER, GREER, THORBECKE, 1984).

No obstante, niuna medida de la pobreza basada en la carencia de alimentos, ni otra basada en las medidas de la desigualdad, permiten establecer cuáles son los activos de los pobres y cómo se utilizan para construir alternativas. En este sentido, la pobreza se presenta bajo un esquema estático de carencias, pero en las cuales los activos son ignorados.

Bebbington (1999), con base en un estudio de caso en los Andes, ubica la cuestión de las capacidades en torno a la disponibilidad de cinco tipos de capital que serían la base para el desarrollo de capacidades y la interacción de los hogares rurales con el Mercado, el Estado y la Sociedad Civil (BEBBINGTON, 1999). Dichas formas de capital son: el natural o territorial, el humano, el físico, el social y el cultural. A los cuatro primeros tipos de capital, reconocidos por el Banco Mundial, añade la cultura, como identidad y arraigo a las formas de vida rural.

Reardon, por su parte, al vincular la pobreza con el medio ambiente, argumenta que hay que definir la pobreza rural en función de los recursos y las elecciones de inversión de las unidades agrícolas. Dichas decisiones están determinadas por los recursos naturales, la situación de las unidades productivas y las condicionantes externas a dichas unidades. Dicho de otra manera, las decisiones pueden incluir el trabajo agrícola o la diversificación del empleo con impactos directos en el medio ambiente. Las decisiones para aumentar las capacidades se encuentran vinculadas a la sustentabilidad ambiental de las unidades rurales (REARDON, VOSTI, 1995). 
Finalmente, De Janvry y Sadoulet al estudiar las nuevas experiencias de desarrollo rural territorial, como una nueva forma de implementar políticas integrales, afirman que son un avance respecto a los planes de desarrollo sectorial que dominaron las políticas de desarrollo rural en los años sesenta, setenta y ochenta. Lo que distingue estas nuevas experiencias es la creciente participación de organizaciones locales en la formulación e implementación de políticas. Los autores desarrollan un esquema en el que se integran las políticas, la implementación de programas, las organizaciones locales y la inversión en diferentes formas de capital (DE JANVRY, SADOULET, 2004, 2005).

De acuerdo a las propuestas anteriores nos interesa mostrar cómo es que estos activos logran jugar un papel dinámico en cuanto los introducimos como recursos territoriales y nos permite que la densificación de los lazos sociales y el medio ambiente aparezcan como elementos a destacar. En la figura 1 podemos observar como los recursos, las políticas y las instituciones juegan un papel fundamental en el bienestar. La demanda de productos rurales típicos de calidad y de servicios está creciendo e incorporándose rápidamente a los condicionantes del desarrollo rural. La elaboración de artesanías, la conservación y certificación del patrimonio arquitectónico y gastronómico, el turismo y la conservación de la biodiversidad, entre otras actividades, se convierten en decisiones relacionadas directamente con el empleo, el ingreso y el bienestar. 


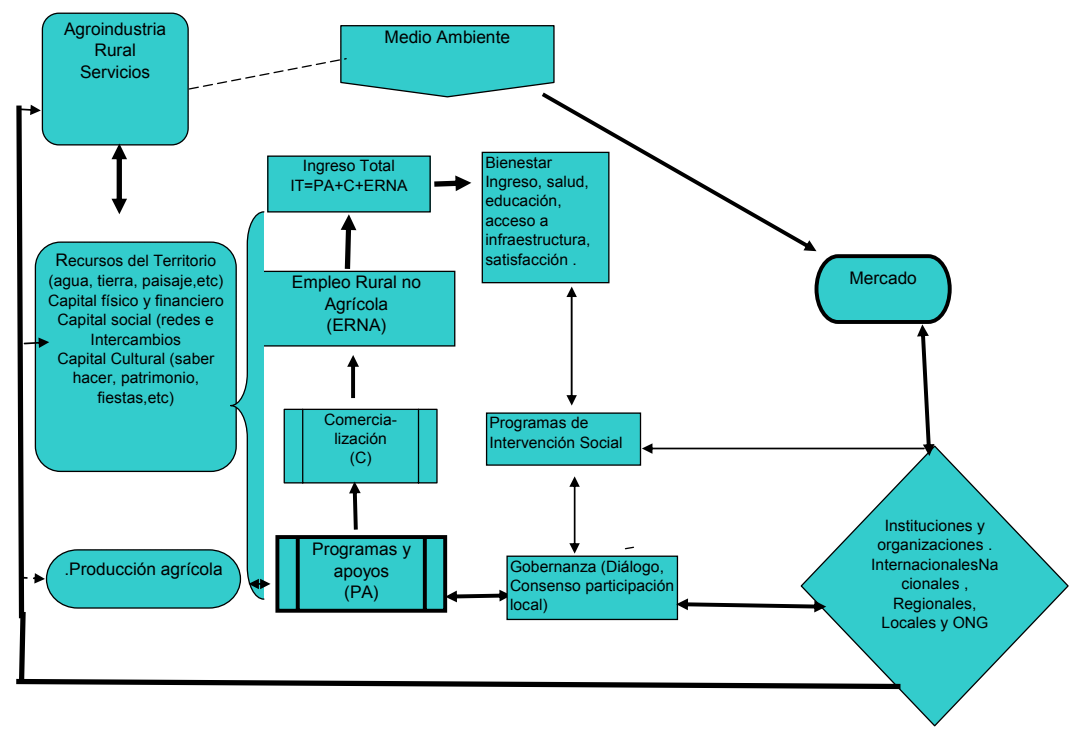

Figura 1 - Propuesta de desarrollo rural territorial, capacidades y multifuncionalidad

La producción agrícola es la actividad fundamental de los hogares campesinos, pero es determinada por los recursos naturales, es decir, el agua, el suelo, el aire, el clima y el paisaje. A este recurso se unen otras formas de capital como son las inversiones previas, la tecnología, las redes, las relaciones sociales y los recursos culturales tales como las fiestas, la participación en actividades colectivas, como el tequio, en caso de Oaxaca, México, y el gusto de los emigrados por financiar obras del pueblo, como la parroquia, las mayordomías de alguna festividad, etcétera. Estos recursos, o formas de capital en la terminología del Banco Mundial y de Bebbington, configuran una estrategia (no en un sentido racional y utilitarista propio de la economía neo-clásica, sino como decisiones más o menos reflexionadas en el contexto de los hogares y de la colectividad) que implica la participación, la interacción con las instituciones gubernamentales y de la sociedad civil que influyen en la hechura de las políticas, de programas específicos de intervención social (becas a poblaciones vulnerables, por ejemplo) y en la gobernanza. Todo ello impacta, como lo hemos visto en la figura anterior, en una mejor calidad de vida. 
Una conclusión del esquema anterior, es la localización regional del uso de los recursos y el desarrollo de las capacidades. Ello nos introduce a la dimensión de pensar el desarrollo rural en términos de capacidades territoriales es decir, en el uso de los recursos y en las decisiones de inversión que pueden redundar en una mayor o menor capacidad de funcionamiento de los hogares y personas en el mercado. De acuerdo a lo anterior, podríamos establecer varias categorías que vinculan la pobreza rural con el mercado de trabajo y con las economías locales.

En la categoría uno, relacionada con la pobreza alimentaria, pero no necesariamente confundida con ella, nos encontraríamos con una pobreza de capacidades en la medida que las elecciones se ven constreñidas por un escaso valor de los recursos territoriales derivados de un deterioro medioambiental o de la lejanía de las localidades de las carreteras y de los centros urbanos. También se caracteriza por la depresión de los recursos humanos, de los saberes tradicionales y un deterioro del patrimonio. En esta categoría se inscriben territorios en los cuales la gente puede estar satisfecha en sus situaciones de carencia y no cree que haya algo necesario para cambiar esa situación. En este ámbito la migración podría ser un activo, pero la carencia de recursos es tal, que la gente no emigra. Estos territorios son excluidos y dependen casi en su totalidad de la intervención del Estado o de agencias no gubernamentales, dado que existe un acelerado deterioro causado por la desinversión en capital social, de activos y de recursos medioambientales.

En la siguiente categoría se inscriben los habitantes de territorios que cuentan (a nivel de sobrevivencia), con mínimos recursos humanos, expresados por la escolaridad y un precario acceso a los servicios de salud, pero con potencialidades de desarrollo por encontrarse en áreas cercanas a las ciudades y por poseer algún producto valorizable para el consumidor urbano o regional, es decir, de agricultura peri-urbana. Estos territorios con capacidades medias son aquellos en los que se realizan tareas mínimas de cuidado del suelo y del agua; existe una retroalimentación incipiente de la migración y muestran potencialidades de inversión.

Los territorios de capacidades más desarrolladas son aquellos en los que las sinergias de los sistemas de mercado han hecho algún efecto y plantean alternativas de elección y de inversión para sus habitantes. Esos 
territorios se inscriben en la lógica de la demanda urbana, pueden tener acceso a tecnologías que incrementen la diferenciación y valorización de los productos y están en proceso de "clusterización" es decir, de aglomeración de empresas agroindustriales inscritas en redes que propician la difusión y el aprendizaje. Adicionalmente, existen formas de recreación cultural que refuerzan la identidad, la autoestima y por tanto, el funcionamiento de las unidades; en suma, se trata de territorios en los cuales la gobernanza se expresa como un proceso de acuerdos, de constitución de derechos y de sinergias institucionales. Sin embargo, la diferenciación de estos territorios, la generación y apropiación de valor de los productos típicos y los sellos de calidad que se imponen a estos productos por parte de los productores y las instituciones regionales y nacionales puede conducir a la exclusión de otros actores. En el ámbito Latinoamericano existen evidencias al respecto, como el caso del Tequila, y los quesos de Cajamarca, Perú (BOUCHER, CARIMENTRAND, REQUIER-DESJARDINS, 2003).

\section{Las capacidades y la generación de economías locales}

El modelo de capacidades para explicar las relaciones entre pobreza rural y mercados de trabajo encuentra un soporte en las críticas que las teorías neoinstitucionalistas han hecho a los modelos clásicos de oferta y demanda, al señalar la inequidad en el acceso a recursos e información y a las disparidades ocasionadas por la capacidad de elección de los productores o trabajadores en el Mercado de Trabajo Rural o la imposibilidad de realizar una elección por la ausencia de información o por las circunstancias. El ejemplo más claro es la satisfacción ante el empleo. El bienestar subjetivo ${ }^{3}$ es en principio un correlato de la libertad y de los logros. No obstante, en el caso de una persona que carezca de alternativas, es probable que se declare satisfecha por la limitación de expectativas, lo que la lleva a restringir al máximo su funcionamiento. Por ello, la satisfacción basada en logros específicos y no la resignación, sugiere un mejor desarrollo de las instituciones y su funcionamiento, así como el de la productividad. En este sentido, la economía y sociología neoinstitucionales (NORTH, 1990; AYALA ESPINO, 2000; PETERS, 1999; YONAY,

\footnotetext{
${ }^{3}$ Entendemos por bienestar subjetivo un indicador de la satisfacción que se deriva con el "estar a gusto" con lo que se
} tiene y se hace. 
1998; WILLIAMSON, 1989; SECKLER, 1977) al insistir en el carácter histórico de la economía y en las "reglas de juego" construidas por los agentes y que configuran los límites a la acción individual han puesto de relieve la construcción de las asociaciones e instituciones sociales como parte del mercado.

Las instituciones y asociaciones permiten un mejor desarrollo de las capacidades y se encuentran en función directa a la superación de la pobreza, porquedisminuyen los costos económicos paralos pobres. Una delas cuestiones más notables de la teoría neoinstitucional y de sus vertientes sociológicas es la importancia que se le ha dado al papel de los costos de transacción ${ }^{4}$ en la constitución de economías de escala. La existencia de marcos institucionales y de asociación permiten disminuir los costos extras que se generan en los intercambios y que se derivan de contratos que implican el establecimiento de una burocracia administrativa especial para dar seguimiento, monitorear y evaluar los productos adquiridos y el cumplimiento de las condiciones de adquisición. El énfasis que ha puesto esta corriente en los llamados "intangibles", imputables a un territorio explica los procesos de desarrollo, que generalmente no es posible comprender cabalmente por medio de los modelos de equilibrio de la oferta y la demanda. Dichos intangibles también operan en las economías locales y se basan en un ethos que distingue a los habitantes de los territorios y que están dados por la cultura. Este ethos se encuentra presente en las redes, las asociaciones y los contratos (muchas veces a la palabra), por medio de las manifestaciones de confianza, que contribuyen a aligerar el peso del funcionamiento institucional, conformando externalidades positivas en los territorios.

En suma, la relación entre mercados de trabajo rural y pobreza incluye una serie de dimensiones entre las cuales el ingreso y el acceso a los satisfactores básicos son una parte de los arreglos, los logros y funcionamientos que se encuentran en función de la apropiación de activos (entre los que los programas de combate a la pobreza son una parte) pero que requieren de la transversalización de valores que refuercen el funcionamiento de las instituciones. En otras palabras, que faciliten la gobernanza de los territorios.

\footnotetext{
${ }^{4}$ Entendidos como los costos extras a la adquisición de una mercancía, tales como las costos de selección, de seguimiento, de supervisión e información en los que deben incurrir los agentes para interactuar en mercados imperfectos, es decir, lejanos al modelo de equilibrio.
} 
De este modo, la complementación de las teorías neoinstitucionales, las de la gobernanza de los territorios rurales y de la formación del capital social, han ańadido otras dimensiones al problema del empleo en las zonas rurales al enfatizar el valor de los productos diferenciados y los intangibles que actúan positivamente en las economías de los productos locales.

El estudio de estas dimensiones se ha convertido en una cuestión vital para comprender la importancia del desarrollo rural y las perspectivas del empleo. En los siguientes apartados propondremos una visión del cambio institucional y la forma en que han emergido los procesos de desarrollo local en la problemática de los mercados de trabajo rural.

\section{El territorio y los mercados alternativos}

Consideramos que para hacer una relación correcta entre mercados rurales y pobreza rural por medio de las capacidades, es posible distinguir las que se identifican con el saber hacer y el dominio de las actividades y los procesos, de las que se relacionan con los valores: tales como la identidad, los lazos de confianza, las redes de enseñanza-aprendizaje y difusión, etcétera. Si partimos de la idea que el territorio es un espacio socialmente construido en el que los recursos localizados son un activo, el desarrollo depende de la existencia de las capacidades. El conocimiento y defensa de los bienes tangibles (infraestructura, disponibilidad de activos físicos, tales como el agua, la calidad del suelo, la cercanía con centros urbanos, etcétera) como de los intangibles (capacidades, objetivas, tales como el saber hacer, y las subjetivas, como las redes de difusión del conocimiento, de intercambios solidarios, de participación social, patrimonio gastronómico, cultura, identidad, etcétera), caracterizan la existencia de territorios con alto potencial de defensa de sus productos y de sus mercados.

Estas dos características han sido enfatizadas por los organismos financieros internacionales, por las instituciones internacionales relacionadas con la agricultura y la alimentación y por los gobiernos de la región que han impulsado recientemente políticas de desarrollo rural de base territorial con el objetivo de fomentar el empleo rural y combatir la pobreza en el campo, aunque dicho sea de paso, estas políticas han sido insuficientes(Schejtman y Berdegué. 2004). 
Las nuevas visiones del desarrollo territorial rural han tenido, desde hace por lo menos dos décadas, un fuerte antecedente en los trabajos sobre la Agroindustria Rural (BOUCHER, 1998) y en el debate sobre el desarrollo local (ALBURQUERQUE, 2004; RAFFESTIN, 1993; VÁZQUEZ BARQUERO, MADOERY, AROCENA, 2001; VÁZQUEZ BARQUERO, 2002).

En el ámbito europeo, las razones para impulsar políticas de desarrollo rural $^{5}$ centradas en el empleo obedecen, entre otras causas, a la pérdida de rentabilidad de la agricultura, la mecanización de los procesos y la pérdida de significación demográfica de la población rural. La Políticas de Agraria Común (PAC) ha transitado por ello desde el subsidio a la producción agrícola a una segunda fase que paulatinamente - en un horizonte que abarca hasta el año 2013-introducirá los apoyos a las actividades rurales no agrícolas y al cuidado medioambiental ${ }^{6}$. Esta segunda fase, que se inscribe en los proyectos denominados Leader ha mostrado sus bondades al propiciar proyectos de desarrollo rural e integración asociativa. Dichos proyectos han reconocido ampliamente los procesos de multifuncionalidad del empleo, la diversificación de la renta y el crecimiento de lo sectores industrial y de servicios en el campo.

En América Latina las particularidades de la región han impedido que los procesos de integración se den bajo los supuestos virtuosos de fomento al asociacionismo. Por el contrario, se han concentrado en la individualización de la producción agropecuaria, profundizando el proceso de desigualdad en cuanto a las capacidades de los territorios privilegiando aquellos que históricamente estaban ya vinculados con el mercado externo.

Las observaciones de los sistemas productivos y las capacidades en América Latina indican que la diferenciación de los recursos no es homogénea. La evaluación de los municipios y de las localidades desde el punto de vista de su infraestructura, de los recursos en capital humano disponible, así como de las relaciones sociales que pueden propiciar la implantación y desarrollo de

\footnotetext{
${ }^{5}$ Schejtman y Berdegué (2004) definen el Desarrollo Territorial Rural como "...un proceso de transformación productiva e institucional en un espacio rural determinado, cuyo fin es reducir la pobreza rural”.

${ }^{6}$ Ello no implica, no obstante, que no existan los fenómenos de exclusión. Tal es el caso de los trabajadores de Europa del Este, pero sobre todo de los inmigrantes magrebíes y subsaharianos que se encuentran en situaciones de exclusión lamentables.
} 
proyectos agroindustriales y de servicios, hablan de la necesidad de impulsar políticas diferenciadas y descentralizadas.

En este contexto, la valoración de las actividades cotidianas relacionadas con la poscosecha y que se encuentran destinadas al autoconsumo o a mercados locales y que tradicionalmente son emprendidas por las poblaciones rurales pueden desempeñar un papel fundamental para la retención de la renta rural, mediante una serie de acciones institucionales entre las que destacan:

- El conocimiento de los productos tradicionales elaborados en un territorio específico por razón de su encadenamiento y de la importancia socio-cultural que esos productos tienen en el consumo;

- El desarrollo de programas destinados a fomentar la calidad y la certificación de los productos.

Para comprender mejor estos fenómenos y sus aportaciones, es preciso ubicar en el ámbito de la teoría los fundamentos de la nueva ruralidad y de esta manera encontrar elementos que permitan reflexionar sobre la importancia de la agroindustria rural para las políticas de empleo.

\section{La air en el debate de los mercados de trabajo}

Desde hace aproximadamente 15 años se ha venido configurando un debate internacional sobre las posibilidades que representan las actividades locales de poscosecha en cuanto a la generación de empleo en el medio rural. Al respecto, han existido esfuerzos consistentes para definir la AIRy diferenciarla de la Agroindustria Tradicional que se encuentra integrada en el marco a la Industria Alimentaria de productos genéricos, es decir, de tipo fordista. Las diferencias entre ambas actividades de transformación de los productos agrícolas primarios, se derivan de las variables referentes a la tipicidad como diferenciación, en contra de la generalidad de los productos de la Industria Alimentaria, al tamańo de la unidad agrícola, la utilización de trabajo asalariado y familiar; de la incorporación de los recursos locales, así como el mercado al que están destinados. Por la naturaleza de los contratos agrícolas, las tendencias actuales marcan una creciente integración y coordinación vertical que en principio está dada por la Industria Alimentaria, pero que muestra una ingerencia cada vez mayor de la Gran Distribución. 
El creciente interés en las potencialidades que representa la AIR se encuentra se deriva de la valoración de los recursos locales, lo que indica, en Latinoamérica, un mercado creciente de productos diferenciados y que al igual que en otras regiones del mundo como en Europa, puede ser la base para procesos de integración horizontal y vertical, de filières o cadenas de producción, transformación y consumo; y por lo tanto de conglomerados o clusters industriales que serían la base para un desarrollo endógeno.

La AIR en Latinoamérica supone la integración de los procesos agroindustriales que implican la posibilidad de incorporar el trabajo agrícola en diversos niveles que van desde la explotación agraria campesina de subsistencia hasta las explotaciones agrarias modernas. Una definición de la AIR es la que ha proporcionado Boucher del Programa Cooperativo de Desarrollo de la Agroindustria Rural en América Latina y el Caribe (PRODAR) del Instituto Interamericano de Cooperación para la Agricultura (IICA). La AIR se define

como la actividad que permite aumentar y retener, en las zonas rurales, el valor agregado de la producción de las economías campesinas, a través de la ejecución de tareas de poscosecha en los productos provenientes de explotaciones silvo-agropecuarias, tales como la selección, el lavado, la clasificación, el almacenamiento, la conservación, la transformación, el empaque, el transporte y la comercialización (BOUCHER, 1998, p. 3).

La generación, pero sobre todo la retención de valor es el elemento clave que distingue a las AIR de las actividades campesinas tradicionales y permite explotar los recursos locales para la construcción de alternativas. Se estima que en América Latina existen 5 millones de unidades empresariales de este tipo, y dentro de ellas, 15 millones de personas que dependen de las actividades de poscosecha (RIVEROS, 2001). Para Boucher (1998), la AIR puede ser tradicional o inducida. Dentro de la primera categoría se inscriben actividades tales como los beneficios primarios de cacao o café, en tanto que la segunda actividad, la inducida, es el resultado de la aplicación de un proyecto de desarrollo que toma en cuenta la especificidad regional y local de los productos y de los procesos que llevan hasta el consumidor final. 
Tomando en cuenta esta última circunstancia, las alternativas del desarrollo rural, el análisis territorial, ha surgido como un elemento indisoluble de la construcción de las AIR para fortalecer la especificidad anteriormente señalada. Los análisis llevaron a clasificar la agroindustria rural de acuerdo a su nivel de desarrollo, como casera, artesanal, semi-industrial. Industrial; y también de acuerdo a criterios de inversión, de calidad, del número de empleados, etcétera.

Finalmente, el análisis de la AIR ha conducido a la consideración de que las actividades en las que se desenvuelve la actividad poscosecha se encuentra relacionada con la existencia de instituciones de crédito, de comercialización y de difusión del saber que actúan en el nivel local disminuyendo los costos de transacción.

De acuerdo a lo anterior, las AIR pueden, en suma, ser una alternativa a la pobreza en la medida que

- Atienden a la especificidad de los territorios, entendidos no sólo como espacio físico sino como el lugar en el que se interrelacionan procesos históricos y culturales

- Movilizan los recursos locales y valorizan los activos de los territorios (incluidos identidad y cultura)

- Tienen una flexibilidad institucional que permite la disminución de costes de transacción; y

- Configuran relaciones complejas de intercambio, de servicios complementarios y de difusión de innovaciones en un contexto local, es decir, en la medida que constituyen un SIAL.

Dado que la teoría ha encontrado una veta en esta área nos referiremos a continuación a este concepto.

\section{Los SIAL ¿Una alternativa viable a la pobreza?}

El estudio y observación de las actividades de transformación de los productos locales ha derivado en la propuesta metodológica del estudio de esas actividades como "sistemas" integrados de producción, transformación y consumo, pero relacionados con el mercado regional y global (REQUIERDESJARDINS, 2006). Las AIR integradas en un SIAL presentan 
posibilidades de coordinación vertical y de integración de cadenas que se constituyen en alternativas de empleo y de acumulación de capital social, de redes de difusión del saber hacer y de innovación que tienden a concentrar actividades agrícolas, industriales, técnicas y culturales en un territorio determinado.

En este sentido se entiende por SIAL "Sistemas constituidos por organizaciones de producción y de servicio (unidades agrícolas, empresas agroalimentarias, empresas comerciales, restaurantes, etcétera) asociadas mediante sus características y su funcionamiento a un territorio específico" (Boucher. 1998). También se les ha visto como un entramado de símbolos que configuran la identidad y que adquieren valor por eso mismo (MOLTYMÄIZY, MUCHNICK, 2005; MUCHNICK, 2004). Para otros autores, el concepto mismo ha tenido una evolución que lo ha llevado a ser considerado como producto y parte del capital social y de la gobernabilidad (COURLET, 2002). La definición del SIAL surge teóricamente de una combinación de la teoría de los sistemas y de la sociología y economía industrial e institucional, que a partir del concepto de "Distrito Industrial" elaborado por Marshall y retomado por Becattini (BECATTINI, 1998, 1999; PYKE, BECATTINI, SENGENBERGER, 1990) en Italia, mostró su poder para describir y analizar las características del desarrollo industrial de las ciudades medias. Las características del distrito, se sintetizan en la ubicación de una serie de empresas e industrias en un territorio específico que establecen contratos y formas de intercambio que favorece la interrelación de los agentes y disminuyen sustancialmente los costes de transacción, porque dichos contratos se dan en un marco de confianza y de ambientes institucionales que favorecen dichos intercambios.

La observación de que las empresas se agrupaban en torno a un producto y seguían pautas determinadas por las instituciones forjadas en dicho territorio dio lugar a que Schmitz (1995) y Porter (1998) avanzaran en la definición de los distritos como "conglomerados" o Clusters que facilitaban el desarrollo endógeno de los territorios.

Finalmente, la observación empírica de que las redes establecidas por los agentes facilitan, mediante los intercambios de confianza la difusión de innovaciones, permitió establecer el concepto de "territorios" que aprenden 
o territorios inteligentes "Learning Regions".

A partir de estos conceptos, la observación empírica de las cadenas de transformación e industrialización de productos destinados generalmente a mercados regionales, sobre la base de conocimientos locales, mostró un comportamiento semejante a los distritos industriales, por lo que fue posible apreciar un comportamiento tipo cluster o sistema (RALLET, 2002; SANZ CAÑADA, 1997). Sin embargo, la novedad del concepto siempre puede introducir algunas confusiones, para lo cual es preciso delimitar las características que posibilitan un SIAL y lo distinguen de las actividades tradicionales o incipientes de transformación de productos locales:

Brevemente, dichas características son:

- La existencia de un producto local que tenga características específicas otorgadas por el territorio (vino, quesos, embutidos, artesanías, etcétera)

- La existencia y consolidación de instituciones que faciliten el desarrollo de la AIR con base en la confianza y los acuerdos

- Prácticas sociales de innovación y difusión

- Capacitación y apoyo tecnológico generados por los productores o por asociaciones de carácter gubernamental o no gubernamental

- Desarrollo y defensa de la calidad, tomando en cuenta el consumo urbano y las exigencias sanitarias (OYARZÚN, 2005; SANZ CAÑADA, MACÍAS VÁZQUEZ, 2005).

El objetivo fundamental de un SIAL, sin embargo, debe relacionarse con la generación de valor, con la valorización de los recursos locales, para lo cual se enfocan hacia la disminución de los costes de transacción, la incorporación de patrones de calidad y de la protección de las denominaciones geográficas y de origen. La constitución de un SIAL o de un sistema potencial - Tequila en Jalisco, integración de sistemas de producción de nopal en Morelos o los quesos de Cotija, en Michoacán, México, - se plantea en última instancia bajo la problemática del consumo urbano y de la valorización creciente de los alimentos tradicionales. Ello apunta a que sean considerados como una posibilidad en la lucha contra la pobreza pero requieren como en los distritos industriales, el desarrollo de capacidades (BOUCHER, CARIMENTRAND, REQUIER-DESJARDINS, 2003), es decir de elecciones y de inversión que se desprenden de un saber hacer con 
los recursos del territorio; una institucionalidad que facilite los procesos de transformación y comercialización de los productos, y de las innovaciones. En este sentido es que los SIAL inducidos por políticas específicas pueden tener éxito en la búsqueda de alternativas a la pobreza rural.

En suma, los SIAL pueden ser sistemas pertinentes para la superación de la pobreza, pero requieren del entorno institucional y de la reafirmación de las características culturales del territorio, además de una decisión empresarial para constituirse como una alternativa, porque también pueden ser una fuente de exclusión de los actores con menores capacidades en un territorio determinado.

\section{Conclusiones}

Hemos abordado el problema de la pobreza rural y lo hemos relacionado con los mercados de trabajo. Al mismo tiempo hemos colocado las capacidades como elecciones en el uso de los recursos de un territorio específico. Las hipótesis que nos planteamos a partir de la revisión de la literatura han permitido establecer grados de correspondencia entre las capacidades y la lucha contra la pobreza rural. La lectura de algunos estudios de caso, sugieren que dichas hipótesis pueden ser exploradas en estudios futuros. Sobre todo, la a partir de la experiencia de la Unión Europea, podemos explotar en América latina las bondades de un marco conceptual que vincule mercados de trabajo, capacidades, territorio y construcción de instituciones.

Esta propuesta es relevante en la medida que puede tener implicaciones de política que atiendan a la búsqueda de alternativas de industrialización de los productos alimenticios o de otro tipo, (prendas de vestir, calzado, etcétera) a diversas escalas: casera, artesanal o industrial. Entre las alternativas que hemos explorado en el ámbito de la industrialización de AL y la posibilidad de constituir políticas de desarrollo endógeno hemos observado la AIR en sus diversas vertientes. Sin embargo, hemos hecho énfasis en que la AIR y las políticas para impulsarla adolecen de graves limitantes si no se les contextualiza en la constitución de Sistemas Agroalimentarios que integren relaciones de coordinación vertical y horizontal. Por ello hemos hecho énfasis en la construcción de políticas regionales y descentralizadas que 
ubiquen los territorios con capacidades y de un ambiente institucional que propicie la gobernanza como elemento fundamental de cualquier política de desarrollo. 


\section{Referencias Bibliográficas}

ALBURQUERQUE, Francisco. Local economic development y decentralization in Latin America. CEPAL, n. 82, p. 155-169, abr. 2004.

APARICIO, Susana; BERENGUER, Paula; RAU, Víctor. Modalidades de intermediación en los mercados rurales de Argentina. Cuadernos de Desarrollo Rural, n. 53, p. 59-79, 2004.

ASTORGA LIRA, Enrique. Mercado de trabajo rural en México: la mercancía humana. México: Era, 1985.

AYALA ESPINO, José. Instituciones y economía. Una introducción al neoinstitucionalismo económico. Mexico, D.F: Fondo de Cultura Económica, 2000.

BARRÓN PÉREZ, M.; SIFUENTES, Emma; PEDRERO, Mercedes. Mercados de trabajo rurales. Estudios de caso y metodologías. México: Tepic, UNAMUAN, 1997.

BEBBINGTON, Anthony. Capital and capabilities: a framework for analyzing peasant viability, rural livelihood and poverty. World Development, v. 27, n. 12, p. 2021-2044, 1999.

BECATTINI, Giacomo. L'Industrializzazione leggera della Toscana: ricerca sul campo e confronto delle idee. Milano: F. Angeli, 1999.

. Distretti industriali e made in Italy: le basi socioculturali del nostro sviluppo economico. Torino: Bollati Boringhieri, 1998.

BOUCHER, François. Agroindustria rural en el horizonte del 2000. Perú: Instituto Interamericano de Cooperación para la Agricultura (IICA), Centro Regional Andino, 1998. 11 p.

BOUCHER, François; CARIMENTRAND, Aurèlie; REQUIERDESJARDINS, Denis. Agroindustrie rurale et lutte contre la pauvrete: les systemes agroalimentaires localises contribuent-ils au renforcement des Capabilités? Ponencia para el 3eme Colloque sur l'Approche des Capacités. Université de Pavie: 2003. 
COMITÉ TÉCNICO DE MEDICIÓN DE LA POBREZA. Medición de la pobreza. Variantes metodológicas y estimación preliminar. México: SEDESOL, 2002.

COURLET, Claude. Les systemes productifs localises. Un bilan de la literatura. Etud. Rech. Syst. Agraires Dev, n. 33, p. 27-40, 2002.

DE JANVRY, Alain; SADOULET, Elizabeth. Achieving success in rural development. Toward an implementation of an integral approach. Agriculture Economics, v. 32, n. sl, p. 75-89, 2005.

. Fitting the facts and capitalizing on new opportunities to redesign rural development programs in Latin America. Revista de Economía e Sociologia Rural, v. 42, n. 31, p. 399-429, Sept. 2004.

FOSTER, James; GREER, Joel; THORBECKE, Erick. A class of descomposable poverty measures. Econometrita, v. 52, n. 3, p. 761-766, mayo. 1984.

GARZA TOLEDO, Enrique de la; SALAS, Carlos. Reestructuración productiva, mercado de trabajo y sindicatos en América Latina. Buenos Aires: Consejo Latinoamericano de Ciencias Sociales, 2000.

LARA FLORES, Sara María. Análisis de los mercados de trabajo rural en México en un contexto de flexibilización. ¿Una nueva ruralidad? Buenos Aires, Argentina: Consejo Latinoamericano de Ciencias Sociales, CLACSO, p. 363-382, 2001.

MOLTY-MÄIZY, Pascal; MUCHNICK, José. Circulation et construction de savoir-faire: questions pour une anthropologie des systemes agroalimentaires localises. Colloque Systèmes Agroalimentaires Localisés: Produits, Enterprises Et Dynamiques Locales-Montpellier, Du 16-18 Octobre 2002. Industries Agroalimentaries Agricoles, p. 16-26, sept. 2005.

MUCHNICK, José. Identidad territorial de alimentos. Alimentar el cuerpo humano y el cuerpo social. Ponencia. Congreso Internacional Arte Agroindustria Rural y Territorio. Toluca, México: 2004.

NORTH, Douglass Cecil. Institutions, institutional change, and economic performance. Cambridge, New York: Cambridge University Press, 1990. 
OYARZÚN, María Teresa. Sellos de calidad en productos alimenticios agroindustriales con perspectiva para la pequeña agroindustria rural en América Latina. Santiago, Chile: Biblioteca Virtual, Proyecto Regional de Cooperación Técnica para la Formación en Economía y Políticas Agrarias y de Desarrollo Rural en América Latina, FODEPAL, 2005.

PETERS, B. Guy. Institutional theory in political science the new institutionalism. London: Continnum, 1999.

PORTER, ME. Clusters and the new economic of competition. Harvard Bussiness Review, v. 76, n. 6, p. 77-90, Nov./Dec. 1998.

PYKE, Frank; BECATTINI, G.; SENGENBERGER, W. Industrial districts and inter-firm co-operation in Italy. Geneva: International Institute for Labour Studies, 1990.

RAFFESTIN, Claude. Por uma geografia do poder. São Paulo, Brasil: Ática, 1993.

RALLET, Alain. Léconomie de proximites. Propos d'Etape. Etud. Rech. Syst. Agraires Dév., n. 33, p. 11-25, 2002.

REARDON, Thomas; VOSTI, Stephen A. Links between rural poverty and the environment in developing countries: asset categories and investment poverty. World Development, v. 23, n. 9, p. 1495-1506, 1995.

REQUIER-DESJARDINS, Denis. Globalización y evolución de la agroindustria rural en los Países Andinos. Un enfoque sobre los "Sistemas Agroalimentarios Localizados”. IICA-PRODAR (database online), 1 junio 2006. Disponible en: http:// www.cybercable.tm.fr/ -jarmah/public_html/ DENIS4.htm\#2.

RIVEROS, Hernando. Agroindustria rural: conceptos, características y oportunidades. Transcripción de la Presentación Oral en el II Curso Internacional Sobre la Promoción de la Agroempresa Rural para el Desarrollo Microregional Sostenible, p. 1-11, 2001. 
SANZ CAÑADA, Javier. Articulación espacial de la cadena agroalimentaria: impacto de los procesos de globalización y reestructuración postfordista. La agricultura familiar en Espańa. Estrategias adaptativas y políticas agropecuarias. Lleida, España: Universitat de Lleida, 1997, p. 149-179.

SANZ CAÑADA, Javier; MACÍAS VÁZQUEZ, Alfredo. Quality certification, institutions and innovation in local agro-food systems: protected designations of origin olive oil in Spain. Journal of Rural Studies, n. 21, p. 476-486, Oct. 2005 .

SCHEJTMAN, Alexander; BERDEGUÉ, Julio A. Desarrollo territorial rural. Santiago de Chile: Centro Latinoamericano para el Desarrollo Rural (RIMISP), 2004.

SCHMITZ, Hubert. Small shoemakers and fordist giants: tale of a supercluster. World Development, v. 23, n. 1, p. 9-33, Jan. 1995.

SECKLER, David William. Thorstein veblen y el institucionalismo. Un estudio de la filosofía social y la economía. México: Fondo de Cultura Económica, 1977.

SEN, Amartya Kumar. Development as freedom. New York: Knopf, 1999. . Nuevo examen de la desigualdad. Madrid: Alianza, 1995.

TORRES SALCIDO, Gerardo. Políticas de empleo y la transformación de los mercados de trabajo en México. Revista Venezolana de Economía y Ciencias Sociales, v. 11, n. 3, p. 127-153, 2005.

VÁZQUEZ BARQUERO, Antonio. Endogenous development: networking, innovation, institutions, and cities. London, New York: Routledge, 2002.

VÁZQUEZ BARQUERO, Antonio; MADOERY, Oscar; AROCENA, José. Transformaciones globales, instituciones y politicas de desarrollo local. Rosario, Argentina: Homo Sapiens Ediciones, Maestría en Desarrollo Económico Local, Universidad Autónoma de Madrid, 2001.

WILLIAMSON, Oliver E. Las instituciones económicas del capitalismo. México: Fondo de Cultura Económica, 1989. 
YONAY, Yuval P. The struggle over the soul of economics: institutionalist and neoclassical economists in America between the wars. Princeton, N.J.: Princeton University Press, 1998. 